\title{
Nghiên cứu tính hiệu quả của việc kết hợp công nghệ BIM và VR trong đào tạo sinh viên chuyên ngành xây dựng
}

\author{
Nguyễn Thanh Bản ${ }^{1}$, Trần Anh Bình" ${ }^{* 1}$, Trần Thu Trang ${ }^{2}$ \\ ${ }^{1}$ Khoa Công nghệ Thông tin, Trường Đại học Xây Dựng \\ ${ }^{2}$ Khoa Công nghệ Thông tin, Trường Đại học Đại Nam \\ TỪ KHOÁ \\ TÓM TẮT
}

Thực tế ảo

Mô hình thông tin công trình

Đào tạo xây dựng

\begin{abstract}
Bài báo tập chung nghiên cứu tính hiệu quả của việc kết hợp công nghệ mô hình thông tin công trình (BIM - Building Information Modelling) và công nghệ thực tế ảo (VR-Virtual Reality) trong đào tạo sinh viên chuyên ngành xây dựng. Cách tiếp cận này giúp cho sinh viên hiểu thiết kế và dữ liệu của mô hình BIM một cách trực quan nhờ sự hỗ trợ của công nghệ VR. Để minh chứng cho sự kết hợp này nhóm tác giả sử dụng phần mềm Autodesk Revit thiết kế mô hình thông tin công trình BIM, Unity 3D Engine xây dựng một ứng dụng thực tế ảo có thể chạy trên thiết bị di động, từ đó lấy ý kiến phản hồi của những sinh viên trực tiếp trải nghiệm.
\end{abstract}

\section{Mở đầu}

Những tiến bộ của khoa học kỹ thuật ngày nay đặc biệt là các công nghệ như thực tế tăng cường, thực tế ảo, phân tích dữ liệu lớn, nhận dạng hình ảnh đã góp phần nâng cao hiệu quả trong công tác quản lý dự án, cả về chi phí lẫn tiến độ. Mô hình thông tin công trình (BIM - Building Information Modeling) được biết đến như một cơ sở dữ liệu thông tin đa chiều đang trở thành tiêu chuẩn cho tất cả các giai đoạn trong lĩnh vực kiến trúc, kỹ thuật và xây dựng (AECArchitectural, Engineering, Construction) [1]. BIM khuyến khích các bên liên quan tham gia và cộng tác để đạt được sản phẩm với chất lượng tốt nhất trong tất cả các giai đoạn của dự án như tạo dựng mô hình 3D cho đến việc dùng mô hình đó trong giai đoạn thiết kế (hồ sơ bản vẽ), thi công (quản lý khối lượng, lập biện pháp, an toàn lao động...) và quản lý tòa nhà (bảo trì các thiết bị cơ điện nước), xuyên suốt vòng đời của công trình.

Thực tế ảo (VR - Virtual Reality) hay còn gọi là thực tại ảo là thuật ngữ miêu tả một môi trường mô phỏng bằng máy tính, môi trường này không tĩnh tại mà có thể phản ứng, thay đổi theo ý muốn (tín hiệu vào) của người sử dụng (thông qua các cử chỉ như lời nói, chạm, vuốt...), điều này xác định một đặc tính chính của VR đó là tương tác thời gian thực. Ngoài khả năng nhìn (thị giác), nghe (thính giác), sờ (xúc giác), các nhà nghiên cứu cũng đã nghiên cứu để tạo các cảm giác khác như ngửi (khứu giác), nếm (vị giác). Những năm gần đây, công nghệ thực tế ảo thu hút sự chú ý ngày càng tăng của nhiều lĩnh vực ứng dụng [2], chẳng hạn như kiến trúc, điện ảnh và giải trí, xác định rủi ro, khoa học y tế, trò chơi điện tử, kỹ thuật, thiết kế đô thị, giáo dục và đào tạo, v.v... VR tạo ra một môi trường mô phỏng và nhập vai được hỗ trợ thông qua các thiết bị như kính thực tế ảo, tai nghe giúp mang mô hình ảo đến ngay trước mắt người dùng. Do đó, VR thường được ứng dụng trong các trường hợp như mô phỏng tình huống rủi ro, thử nghiệm giải pháp hay đào tạo luyện tập trước khi sử dụng thực tế (Hình 1).

Là một trong những công nghệ đột phá, VR đã được thử nghiệm và ứng dụng để giải quyết các thách thức trong nhiều dự án ngành $\mathrm{AEC}$ [3]. Những thách thức này bao gồm ra quyết định trong quá trình thiết kế, lập kế hoạch, phát hiện xung đột, đào tạo về an toàn. Nghiên cứu về ứng dụng công nghệ VR trong các giai đoạn khác nhau của các dự án $\mathrm{AEC}$ đã cho thấy những lợi ích mà ứng dụng này có thể mang lại [4]. Công nghệ VR mang lại hiệu quả trong việc kiểm soát tiến độ dự án [5] và đào tạo an toàn xây dựng [6]. Nó cũng có thể xác định các vấn đề thiết kế [7] để giúp người dùng hiểu được độ phức tạp của dự án, cải thiện thiết kế, hỗ trợ việc ra quyết định [3]. Công nghệ VR cung cấp môi trường để cộng tác nhiều hơn giữa những người tham gia dự án [8] và cho phép hiểu rõ hơn về các thiết kế phức tạp ngay ở giai đoạn đầu [9]. Chính sự phức tạp của các dự án xây dựng lớn dẫn đến các sinh viên cần được đào tạo và trang bị kỹ lưỡng những kiến thức cần thiết, sinh viên cần phải hiểu quy trình, thiết bị, nền tảng và phần mềm được sử dụng trong các dự án thực tế. Việc phát triển các ứng dụng BIM và VR có thể giúp giải quyết nhiều vấn đề giáo dục và đào 
tạo [10]. Một nghiên cứu của 5 tạp chí và hội thảo quốc tế đã cung cấp bằng chứng cho thấy việc sử dụng công nghệ VR trong môi trường đào Bảng 1.

Những lợi ích mà công nghệ VR mang lại cho người học. tạo có rất nhiều lợi ích. Bảng 1 dưới đây trình bày tóm tắt kết quả của các nghiên cứu đánh giá lợi ích của VR mang lại.

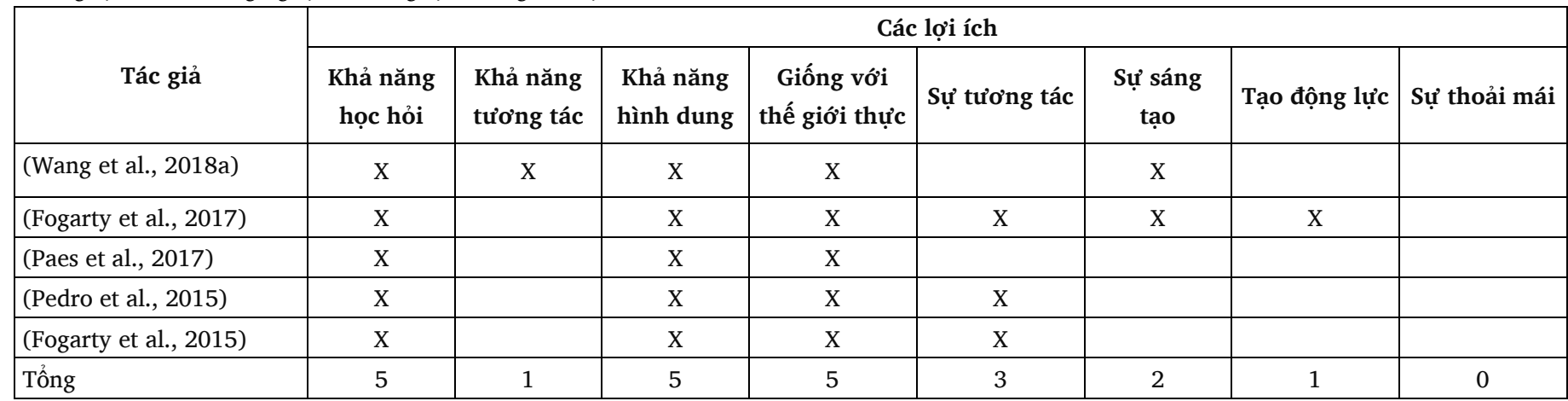

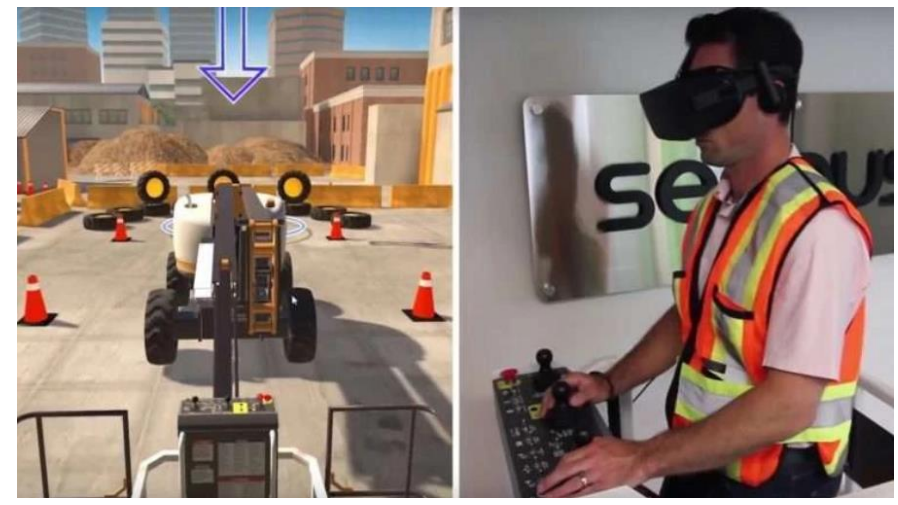

Hình 1. Học viên được đào tạo sử dụng máy xây dựng dựa trên công nghệ VR.

Trong đó: Khả năng học hỏi (Learnability) là dễ dàng học hỏi kiến thức; Khả năng tương tác (Interoperability) là dễ dàng tương tác với các đối tượng 3D trong môi trường ảo; Khả năng hình dung, trực quan (Visualization); Giống với thế giới thực (Real World) cung cấp một mô phỏng giống thế giới thật; Sự tương tác (Interaction) khả năng trao đổi, tương tác với giảng viên và các bạn sinh viên trong nhóm; Sự sáng tạo (Creativity) nâng cao khả năng sáng tạo; Tạo động lực (Motivating) học; Sự thoải mái (Comfort) dễ dàng sử dụng công nghệ.

Do đó, bài báo nghiên cứu những hiệu quả của sự kết hợp giữa công nghệ BIM và VR trong công tác đào tạo chuyên ngành xây dựng, tổng hợp quy trình kết hợp giữa hai công nghệ này. Cuối cùng, nhóm tác giả cũng xây dựng thử nghiệm một ứng dụng chạy trên thiết bị di động giúp sinh viên trải nghiệm không gian phòng 410A1, trường Đại học Xây Dựng trong môi trường ảo, di chuyển xung quanh và tương tác với các vật dụng nội thất trong căn hộ, thay đổi màu sơn tường, màu sơn nội thất, xem thêm các thông tin mô hình BIM. Từ đó khảo sát mức độ hài lòng của sinh viên để làm căn cứ quyết định trước khi áp dụng vào công tác đào tạo.

\section{Quy trình kết hợp công nghệ BIM và VR}

Ngày nay BIM được ứng dụng như là tiêu chuẩn cho các dự án AEC trong hầu hết các công ty xây dựng. Quyết định số 2500/QĐ-TTg ngày 22/12/2016 của Chính phủ về việc phê duyệt đề án nghiên cứu lộ trình áp dụng BIM trong xây dựng; Quyết định 203/QĐ-BXD ngày 21/3/2017 của Bộ Xây dựng về việc thành lập Ban Chỉ đạo thực hiện đề án áp dụng mô hình thông tin công trình (BIM) trong hoạt động xây dựng và quản lý vận hành công trình (gọi tắt là Ban Chỉ đạo BIM) cho thấy việc nghiên cứu ứng dụng BIM trong xây dựng là rất cần thiết. Tuy nhiên, hầu hết các công ty chưa sử dụng công nghệ VR. Do đó, việc ứng dụng công nghệ VR cùng với BIM cần theo một quy trình chặt chẽ, kèm theo sử dụng các thiết bị chuyên dụng. Hình 2 dưới đây mô tả quy trình kết hợp công nghệ BIM và VR để tạo ra một sản phẩm hoàn chỉnh, cụ thể là ứng dụng chạy trên thiết bị di động, phù hợp với đối tượng sử dụng là các kỹ sư hoặc người dùng cuối. Quy trình này cũng có thể áp dụng cho những dự án đã ứng dụng BIM và muốn tích hợp công nghệ VR, cũng như giúp sinh viên tham khảo được kết hợp cần thiết để bắt đầu một đồ án môn học.

Quy trình kết hợp này chia làm 3 giai đoạn. Giai đoạn 1 : Đội ngũ thiết kế sẽ sử dụng các phần mềm thiết kế $3 \mathrm{D}$ chuyên ngiệp như: Revit, 3D Max, SketchUp, AutoCad thiết kế mô hình 3D công trình, căn nhà, việc này được trao đổi thường xuyên với đội ngũ lập trình. Sau đó, họ có thể lựa chọn kết xuất ra định dạng.FBX, đóng gói kèm các vật liệu (Texture) và chuyển giao cho đội ngũ lập trình. Bằng các công cụ lập trình như Unity3D hay UnReal, sẽ thiết lập môi trường VR, xử lý các sự kiện tương tác của người dùng trên mô hình và hoàn thiện sản phẩm, quá trình này gọi là giai đoạn lập trình (giai đoạn 2). Sau đó ứng dụng được cài đặt trên điện thoại di động và trải nghiệm thông qua kính thực tế ảo như: HTC Vive, Oculus Rift, GearVR. (giai đoạn 3)

Ở giai đoạn 2 , một kiến thức quan trọng cần phải nắm được đó khi đưa mô hình BIM vào môi trường VR đó là một hệ thống VR gồm 5 thành phần: phần mềm (Software), phần cứng (Hardware), mang liên kết, người dùng và ứng dụng. Trong đó 3 thành phần chính quan trọng nhất là phần mềm, phần cứng và ứng dụng. 

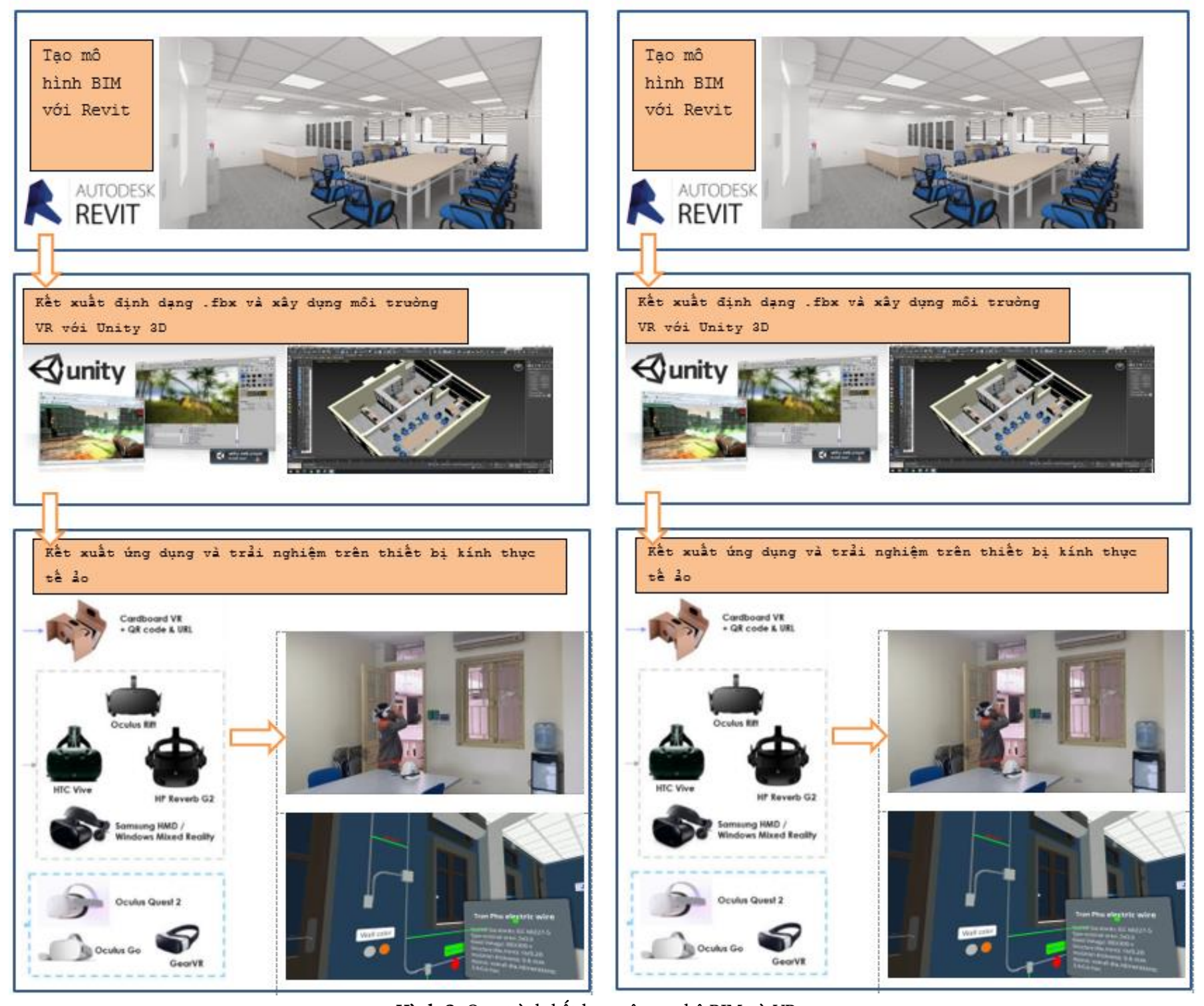

Hình 2. Quy trình kết hợp công nghệ BIM và VR.

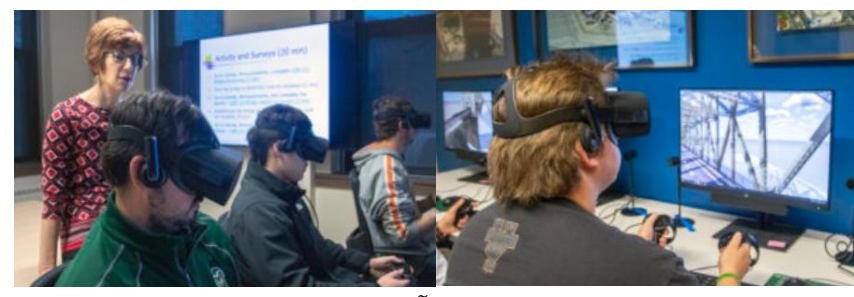

Hình 3. Olbina hướng dẫn sinh viên sử dụng công nghệ VR trong bài giảng của mình.

Phần mềm: phần mềm của hệ thống VR có 2 công dụng chính là: tạo hình và mô phỏng. Các đối tượng của VR được mô hình hóa từ các mô hình 3D sau đó được mô phỏng động học, động lực học và mô phỏng xử lý của đối tượng, xử lý tương tác người dùng.

Phần cứng: phần cứng của hệ thống VR bao gồm: Máy tính có cấu hình đồ họa mạnh mẽ, các thiết bị đầu vào (input devices) và các thiết bị đầu ra (output devices).
- Các thiết bị đầu vào (Input devices): bao gồm những thiết bị đầu ra có khả năng kích thích các giác quan để tạo nên cảm giác về sự hiện hữu trong thế giới ảo. Chẳng hạn như màn hình đội đầu HMD, chuột, các tai nghe âm thanh nổi - và những thiết bị đầu vào có khả năng ghi nhận nơi người sử dụng đang nhìn vào hoặc hướng đang chỉ tới, như thiết bị theo dõi gắn trên đầu (head-trackers), găng tay hữu tuyến (wiregloves).

- Các thiết bị đầu ra (Output devices): gồm hiển thị đồ họa (như màn hình, HDM,..) để nhìn được đối tượng 3D. Thiết bị âm thanh (loa) để nghe được âm thanh vòm (như Hi-Fi, Surround,..). Bộ phản hồi cảm giác (Haptic feedback như găng tay,...) để tạo xúc giác khi sờ, nắm đối tượng. Bộ phản hồi xung lực (Force Feedback) để tạo lực tác động như khi đạp xe, đi đường xóc, ... [11]

Ứng dụng: ứng dụng sau đó sẽ được mô phỏng trên máy tính hoặc các thiết bị thông minh cấu hình cao giúp người dùng trải nghiệm một cách mượt mà như đang đắm chìm trong môi trường ảo. 


\section{Lợi ích của công nghệ BIM và VR trong đào tạo lĩnh vực xây dựng}

Việc chuẩn bị sớm cho sinh viên có được những kiến thức về công nghệ đóng vai trò quan trọng cho đội ngũ nhân lực chất lượng cao trong lĩnh vực xây dựng sau này. Họ có thể trở thành những người định hướng, giải quyết những vấn đề và thách thức trong các dự án lớn. Nhiều nhà nghiên cứu đã nêu tầm quan trọng của giáo dục dựa trên BIM trong các chương trình liên quan đến AEC. Sacks và Barak [12] đã thay thế đồ họa kỹ thuật dân dụng truyền thống các khóa học với BIM cho sinh viên năm nhất. Azhar và cộng sự [13] đã kiểm tra tính hiệu quả hướng dẫn BIM bằng cách khảo sát nhận thức của học sinh. Họ đã chứng minh cách BIM nâng cao hiểu biết của sinh viên về quản lý dự án xây dựng. Trong một nghiên cứu khác, Wong et al. [14] đã nghiên cứu tình trạng, điểm yếu, điểm mạnh và khả năng chuyên môn của giáo dục dựa trên BIM. Peterson et al. [15] đã thêm BIM vào các khóa học quản lý dự án xây dựng của họ và chỉ ra cách BIM có thể hỗ trợ và tăng cường hiểu biết của học sinh. Abdirad và Dossick [16] đề xuất rằng BIM là một môn học cần thiết được giảng dạy ở trường đại học vì nhu cầu của ngành và vì việc phát triển các chiến lược để cung cấp BIM là phức tạp. Một minh chứng nữa đó là vào hè năm 2018, đại học bang Colorado, Mỹ (Colorado Stage University - CSU) đã tài trợ cho giáo sư Olbina và nhóm nghiên cứu của cô 5 bộ dụng cụ VR nhằm mang công nghệ thực tế ảo hỗ trợ công tác đào tạo của trường, đặc biệt chú trọng cho sinh viên ngành kỹ thuật. Các sinh viên được giao nhiệm vụ đi qua một mô hình cây cầu trong môi trường ảo, và nhiệm vụ của họ là tìm kiếm các sai sót trong thiết kế. Olbina mong muốn thông qua việc sử dụng công nghệ VR không chỉ giúp sinh viên hiểu sâu hơn về quy trình xây dựng, mà còn giúp họ phát hiện ra những vấn đề trong một dự án tốt hơn khi nhìn vào bản vẽ 2D.

Như vậy có thể thây rằng, việc kết hợp giữa BIM và VR trong công tác đào tạo có thể mang lại một số lợi ích nhất định cho sinh viên chuyên ngành xây dựng như:

- Tạo cho sinh viên sự tò mò, hứng thú khi tiếp cận các công nghệ mới, từ đó nâng cao hiệu quả truyền đạt kiến thức so với cách dạy truyền thống.

- Tăng khả năng tương tác giữa các sinh viên trong nhóm khi thực hiện chung một dự án/đồ án, họ dễ dàng thử nghiệm các phương án thiết kế khác nhau trong môi trường ảo, từ đó đánh giá được tính hiệu quả của những giải pháp đó, điều mà thực tế khó có thể làm được.

- Giảng viên có thể phản hồi gần như ngay lập tức với sinh viên trên mô hình BIM trong không gian ảo.

- Trải nghiệm trực quan mô hình BIM, giúp sinh viên tiếp cận và hiểu được những khái niệm phức tạp một cách hiệu quả.

\section{Xây dựng ứng dụng thử nghiệm}

Nhóm tác giả dựng mô hình BIM phòng 410A1 trường Đại học Xây Dựng trên phần mềm Revit, kết xuất ra định dạng. FBX, sau đó đưa phần mềm Unity $3 \mathrm{D}$ để lập trình và mô phỏng môi trường VR, các sự kiện như chạm thay đổi màu sơn, chạm trên đường điện, máy chiếu, di chuyển xung quanh mô hình phòng được nhóm xử lý bằng ngôn ngữ lập trình. Unity 3D là một nền tảng hỗ trợ rất mạnh mẽ cho các tác vụ như dựng hình (kết xuất đồ họa) cho các hình ảnh $2 \mathrm{D}$ hoặc $3 \mathrm{D}$, công cụ vật lý (tính toán và phát hiện va chạm), âm thanh, mã nguồn, hình ảnh động, trí tuệ nhân tạo, phân luồng, tạo dòng dữ liệu xử lý, quản lý bộ nhớ, dựng ảnh đồ thị và kết nối mạng, ứng dụng hoàn thiện có thể chạy trên đa nền tảng như: PlayStation 3 , Xbox 360, Wii U, iOS, Android, Windows, Blackberry $10, \mathrm{OS} \mathrm{X}$, Linux, trình duyệt Web và cả Flash. Dưới đây là một vài hình ảnh kết quả của ứng dụng chạy trên thiết bị di động, kính thực tế ảo Bobo VR Z6. Người sử dụng có thể di chuyển tới nhiều góc trong phòng, xem thông tin nội thất căn hộ, đổi màu sơn tường, bàn ghế, nội thất, xem thông tin về các thiết bị điện nước ẩn sau bức tường.

Chúng tôi áp dụng kỹ thuật nạp chồng mô hình (model overloading) khi người dùng chạm vào icon màu đỏ để xem đường dây diện ẩn sau bước tường, cung cấp đúng vị trí của bản thiết kế và thông tin kỹ thuật của thiết bị. Hình 4 (bên trái) mô tả bức tường thực tế, hình bên phải là mô hình kèm đường điện trong môi trường VR khi người dùng đeo kính thực tế ảo. Để tương tác trong môi trường VR, cần hiển thị 1 điểm nhỏ (dot) trên giao diện, sau đó tương ứng với những object cần tương tác, điểm này sẽ được thay đổi thành 1 vòng tròn (ring), chúng được gọi là "reticles" (Hình 4 trên cùng). Nhóm tác giả sử dụng Mobile VR Interaction Pack, một module giúp cho việc xử lý sự kiện chạm (touch) của người dùng lên các đối tượng trong VR được thuận tiện hơn. Hàm process dưới đây sẽ xác định đối tượng cần xử lý sự kiện (như các icon đổi màu sơn nột thất, các thiết bị trong căn hộ như: máy chiếu, máy tính để bàn, cây nước lọc...), di chuyển reticle và chuyển đổi trạng thái từ "dot" sang "ring", khi người dùng touch trên kính thực tế ảo, trạng thái ring sẽ phát ra sự kiện.

Ứng dụng đã được nhóm tác giả thử nghiệm và cho hoạt động tốt trên các thiết bị có cấu hình tối thiểu như sau: (1) màn hình kích thước 5 inches; (2) 2 GB RAM; (3) Qualcomm Snapdragon 410 CPU processor, Quad-core 1.2 GHz; (4) hệ điều hành Android 5.0, iOS 9.0; (5) 8 MP camera. Ngoài ra, chúng tôi cũng cho các sinh viên trải nghiệm và khảo sát ý kiến phản hồi từ họ nhằm có được cách nhìn khách quan. Bộ câu hỏi của chúng tôi tập chung vào một số yếu tố sau (1) hiểu biết về công nghệ $\mathrm{BIM}$ và $\mathrm{VR},(2)$ sự hài lòng khi trải nghiệm và tương tác với mô hình $\mathrm{BIM}$ trong môi trường $\mathrm{VR}$, (3) cảm nhận khi sử dụng thiết bị kính thực tế ảo, (4) BIM + VR giúp sinh viên tăng khả năng sáng tạo trong học tập, (5) khả năng sẵn sàng học hỏi và ứng dụng thực tế, (6) $\mathrm{BIM}+\mathrm{VR}$ có giúp tăng hiệu quả làm việc nhóm. Bảng 2 dưới đây là thống kê kết quả khảo sát 50 sinh viên chuyên ngành xây dựng.

Dựa vào kết quả khảo sát có thể thấy rằng, phần đa sinh viên cho biết họ cảm thấy hứng thú khi trải nghiệm công nghệ này, và nó hoàn toàn khác so với những gì họ thường thấy trên mô hình BIM thuần túy, ứng dụng giúp họ hiểu và xem được thiết kế trực quan hơn, tuy nhiên phản hồi cũng cho thấy sử dụng kính thực tế ảo trong thời gian dài là khá khó chịu. 


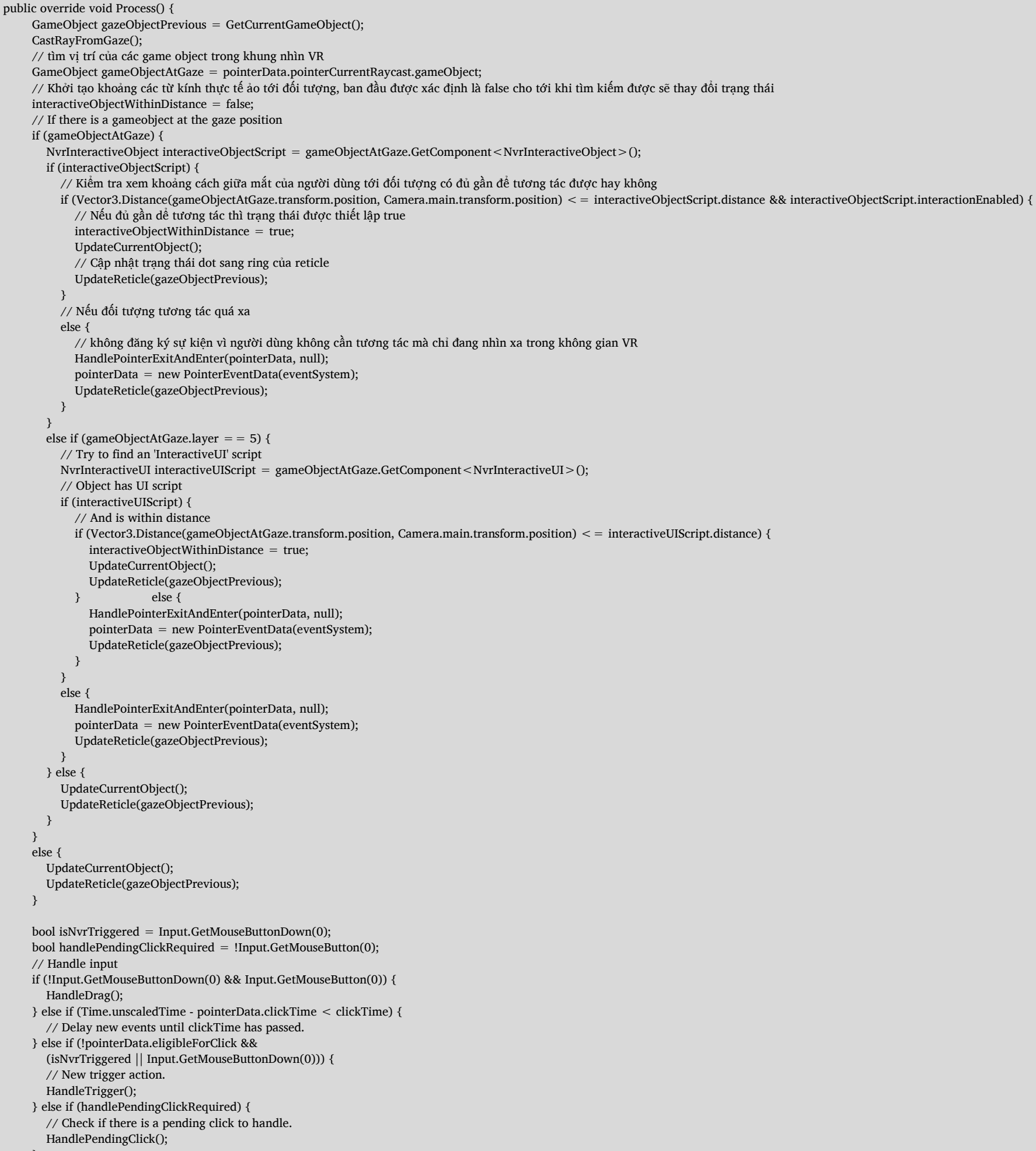




\section{Bảng 2.}

Kết quả khảo sát sinh viên sau khi trải nghiệm ứng dụng kết hợp công nghệ BIM và VR.

\begin{tabular}{|c|c|c|c|c|c|c|}
\hline \multirow{2}{*}{$\begin{array}{l}\text { Câu } \\
\text { hỏi }\end{array}$} & \multirow{2}{*}{ Nội dung hỏi } & \multicolumn{5}{|c|}{ Mức động hài lòng/đồng ý } \\
\hline & & 1 & 2 & 3 & 4 & 5 \\
\hline 1 & $\begin{array}{l}\text { Bạn đã từng biết đến BIM và công } \\
\text { nghệ VR trước đây? }\end{array}$ & 20 (chưa biết) & $\begin{array}{l}5 \text { (đã được nghe về } \\
\text { các công nghệ này) }\end{array}$ & 8 (chỉ biết về BIM) & 7 (chỉ biết về VR) & $\begin{array}{l}10 \text { (đã biết về } \\
\text { BIM và VR) }\end{array}$ \\
\hline 2 & $\begin{array}{l}\text { Bạn có thấy hài lòng khi trải nghiệm } \\
\text { ứng dụng, các tương tác có dễ dàng? }\end{array}$ & $\begin{array}{l}0 \text { (không hài } \\
\text { lòng) }\end{array}$ & 0 (chưa hài lòng) & 10 (hài lòng) & 13 (rất hài lòng) & $\begin{array}{l}27 \text { (cực kỳ hài } \\
\text { lòng) }\end{array}$ \\
\hline 3 & $\begin{array}{l}\text { Kính thực tế ảo khi sử dụng có làm } \\
\text { bạn khó chịu? }\end{array}$ & $\begin{array}{l}5 \text { (không khó } \\
\text { chịu) }\end{array}$ & 12 (khá khó chịu) & 13 (khó chịu) & 5 (rất khó chịu) & $\begin{array}{l}15 \text { (rất khó chịu, } \\
\text { chóng mặt) }\end{array}$ \\
\hline 4 & $\begin{array}{l}\text { BIM + VR có giúp bạn tăng khả năng } \\
\text { sáng tạo trong học tập? }\end{array}$ & 0 (không) & 0 (trung bình) & 7 (cảm nhận có) & 13 (khá sáng tạo) & 30 (rất sáng tạo) \\
\hline 5 & $\begin{array}{l}\text { Bạn có sẵn sàng học công nghệ BIM } \\
\text { và VR, và ứng dụng trong công việc } \\
\text { thực tế? }\end{array}$ & 0 (không) & 5 (cân nhắc) & 15 (sẽ học) & $\begin{array}{l}20 \text { (chắc chắn } \\
\text { học) }\end{array}$ & $\begin{array}{l}10 \text { (đã học và sẽ } \\
\text { ứng dụng) }\end{array}$ \\
\hline
\end{tabular}

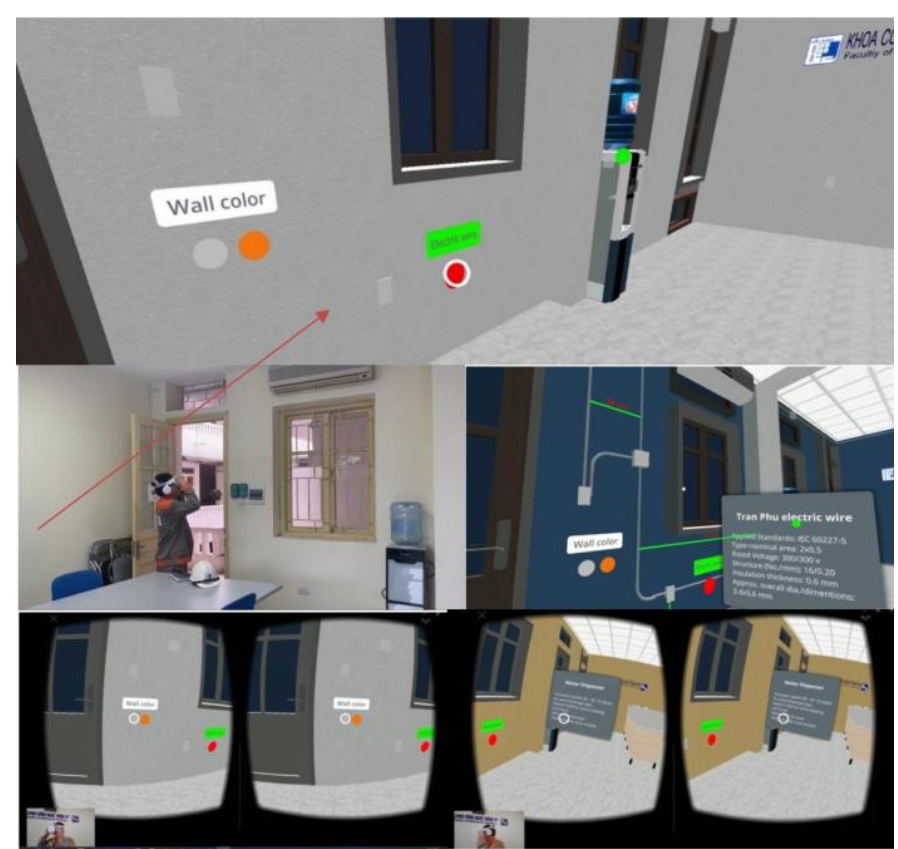

Hình 4. Sinh viên trải nghiệm mô hình BIM qua ứng dụng thực tế ảo VR do nhóm tác giả xây dựng.

\section{Kết luận}

Bài báo đã nghiên cứu và tổng hợp những hiệu quả của việc kết hợp công nghệ BIM và VR trong công tác đào tạo, đặc biệt dành cho sinh viên chuyên ngành xây dựng. Thực tế cũng cho thấy rằng sự kết hợp này giúp sinh viên dễ hình dung về thiết kế hơn, từ đó giúp họ hiểu về dữ liệu và mục tiêu của dự án. Nhóm tác giả cũng xây dựng một ứng dụng VR cho mô hình phòng 410A1. Sinh viên đã trải nghiệm và có những phản hồi tích cực thể hiện ở bảng 2 . Như vậy, để công tác đào tạo nguồn nhân lực ngành xây dựng tiếp cận hiệu quả công nghệ VR, bên cạnh nhà trường thì các doanh nghiệp tuyển dụng nhân sự ngành này cũng là một yếu tố vô cùng quan trọng, nếu doanh nghiệp đẩy mạnh việc ứng dụng VR phục vụ cho hoạt động và kinh doanh thì đó chính là yếu tố thúc đẩy sinh viên có thêm động lực tìm hiểu và nghiên cứu. Các công ty tiếp cận và ứng dụng VR sẽ tạo ra nhiều nhu cầu việc làm cần nguồn nhân lực có kiến thức chuyên môn và công nghệ làm cầu nối giữa nhà trường và doanh nghiệp. Do đó, các công ty/doanh nghiệp có thể kết hợp với nhà trường, bước đầu tổ chức các buổi hội thảo cho thấy tầm quan trọng của việc ứng dụng công nghệ này, đánh giá những lợi ích mà VR mang lại cho doanh nghiệp, bước tiếp theo là có thể hỗ trợ nhà trường thông qua các gói đầu tư và ngược lại phía nhà trường cũng cam kết về chất lượng đào tạo và ký kết những cơ hội việc làm sau khi ra trường dành cho các sinh viên đủ điều kiện đáp ứng nhu cầu công việc của doanh nghiệp đó. Sự kết hợp này mang lại lợi ích cho cả ba bên: nhà trường - doanh nghiệp - sinh viên.

\section{Lời cảm ơn}

Nghiên cứu này được tài trợ bởi Bộ Giáo dục và Đào tạo trong khuôn khổ đề án mã số CT.2019.03.07.

\section{Tài liệu tham khảo}

[1]. Karji, A.; Woldesenbet, A.; Rokooei, S. "Integration of Augmented Reality, Building Information Modeling, and Image Processing in Construction Management: A Content Analysis". In AEI 2017: Resilience of the Integrated Building; American Society of Civil Engineers: Reston, VA, USA, 2017; pp. 983-992.

[2]. Noghabaei, M.; Asadi, K.; Han, K. "Virtual manipulation in an immersive virtual environment: Simulation of virtual assembly". In Proceedings of the Computing in Civil Engineering 2019, Atlanta, Georgia, 17-19 June 2019; pp. 95-102.

[3]. Chen, H.; Hou, L.; Zhang, G.K.; Moon, S. "Development of BIM, IoT and AR/VR technologies for fire safety and upskilling". Autom. Constr. 2021, 125, 103631.

[4]. Pratama, L.A.; Dossick, C.S. "Workflow in Virtual Reality Tool Development for AEC Industry. In Advances in Informatics and Computing in Civil and 
Construction Engineering”; Springer: Berlin/Heidelberg, Germany, 2019; pp. 297-306.

[5]. Fu, M.; Liu, R. "The Application of Virtual Reality and Augmented Reality in Dealing with Project Schedule Risks". In Proceedings of the Construction Research Congress 2018, New Orleans, LA, USA, 2-4 April 2018; pp. 429438.

[6]. Getuli, V.; Capone, P.; Bruttini, A.; Isaac, S. "BIM-based immersive Virtual Reality for construction workspace planning: A safety-oriented approach". Autom. Constr. 2020, 114, 103160.

[7]. Alizadehsalehi, S.; Hadavi, A.; Huang, J.C. "Virtual reality for design and construction education environment”. In AEI 2019: Integrated Building Solutions-The National Agenda; American Society of Civil Engineers: Reston, VA, USA, 2019; pp. 193-203.

[8]. Balali, V.; Noghabaei, M.; Heydarian, A.; Han, K. "Improved stakeholder communication and visualizations: Real-time interaction and cost estimation within immersive virtual environments". In Proceedings of the Construction Research Congress 2018, New Orleans, LA, USA, 2-4 April 2018; pp. 522-530.

[9]. Ahmed, S. "A review on using opportunities of augmented reality and virtual reality in construction project management". Organ. Technol. Manag. Constr. Int. J. 2018, 10, 1839-1852.

[10]. Alizadehsalehi, S.; Hadavi, A.; Huang, J.C. "BIM/MR-Lean construction project delivery management system". In Proceedings of the 2019 IEEE Technology \& Engineering Management Conference (TEMSCON), Atlanta, GA, USA, 12-14 June 2019; pp. 1-6.

[11]. Accessed on: May 17th, 2020. [Online]. Available: https://www.smlease.com/entries/technology/what-is-virtual-reality/.

[12]. Sacks, R.; Barak, R. "Teaching building information modeling as an integral part of freshman year civil engineering education". J. Prof. Issues Eng. Educ. Pract. 2010, 136, 30-38.

[13]. Azhar, S.; Sattineni, A.; Hein, M. "BIM undergraduate capstone thesis: Student perceptions and lessons learned". In Proceedings of the 46th ASC Annual Conference, Boston, MA, USA, 7-10 April 2010.

[14]. Wong, K.-D.A.; Wong, F.K.; Nadeem, A. "Building information modelling for tertiary construction education in Hong Kong”. Electron. J. Inf. Technol. Constr. 2011, 16, 467-476.

[15]. Peterson, F.; Hartmann, T.; Fruchter, R.; Fischer, M. “Teaching construction project management with BIM support: Experience and lessons learned". Autom. Constr. 2011, 20, 115-125.

[16]. Abdirad, H.; Dossick, C.S. "BIM curriculum design in architecture, engineering, and construction education: A systematic review". J. Inf. Technol. Constr. (ITcon) 2016, 21, 250-271. 